\title{
Evaluating Superhydrophobic Surfaces under External Pressures using Quartz Crystal Microbalance
}

\author{
Hamed Esmaeilzadeh ${ }^{1}$, Keqin Zheng ${ }^{2}$, Carol Barry ${ }^{2}$, Joey Mead ${ }^{2}$, Majid Charmchi ${ }^{1}$, Hongwei \\ Sun ${ }^{3, *}$ \\ ${ }^{1}$ Department of Mechanical Engineering \\ ${ }^{2}$ Department of Plastics Engineering \\ University of Massachusetts Lowell, Lowell, MA, 01854, USA \\ ${ }^{3}$ Department of Mechanical and Industrial Engineering \\ Northeastern University, Boston, MA, 02115, USA \\ *Corresponding Author (ho.sun@northeastern.edu)
}

\section{Supplementary Information}

\section{1) Analytical Study of Frequency Response of QCM with Non-slip Surface}

When one of the QCM surfaces comes into contact with a Newtonian liquid, a vibrating flow field is generated in the liquid near the oscillating surface in the direction parallel to the oscillation direction-i.e., the $x$-direction in Figure 1S. The velocity of the liquid is the highest near the surface and rapidly damped to zero due to the viscous dissipation. It was also seen as the wave generated in the QCM penetrates into the liquid.

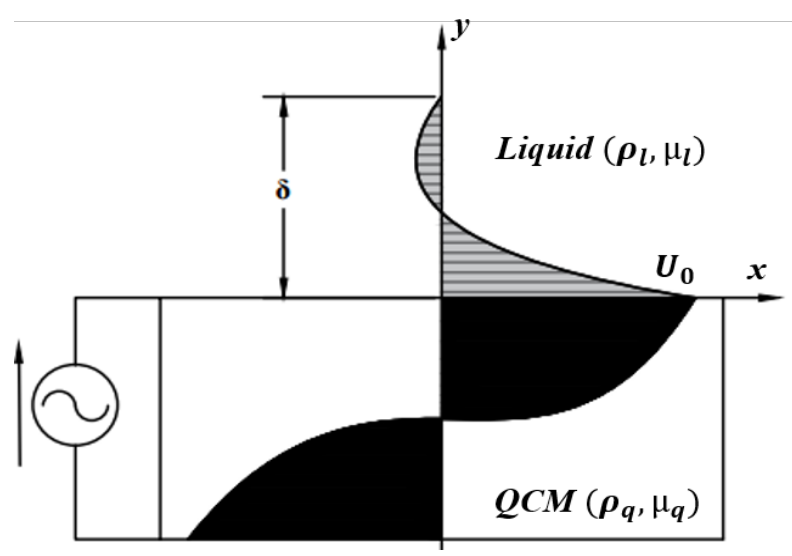

Figure 1S. Cross-section view of QCM with liquid layer loading.

*Corresponding Author: ho.sun@northeastern.edu 
To derive the velocity distribution in the liquid environment, the Navier-Stokes equation is employed. Since the velocity is for one-dimensional plane-parallel flow, the N-S equation is written as:

$$
\frac{\partial V}{\partial t}=v \frac{\partial^{2} V}{\partial y^{2}}
$$

where $\mathrm{V}$ is the velocity of liquid in the $x$-direction, $\mathrm{t}$ is the time and $\mathrm{U}$ is the kinematic viscosity. The initial and boundary conditions for the non-slip condition are as follows:

$$
\begin{aligned}
& V(y, t=0)=0 \\
& V(y=0, t)=\bar{U}_{0} e^{i \omega t} \\
& V(y \rightarrow \infty, t)=0
\end{aligned}
$$

where $\bar{U}_{0}$ is the surface velocity and $\omega$ is the angular frequency of oscillation. Considering the oscillatory boundary condition in equation (3), the velocity of liquid can be written as:

$$
V(y, t)=e^{i \omega t} \varphi(y)
$$

where $\varphi(y)$ is the function of $y$. Based on boundary conditions in equations (3-4), two boundary conditions can be extracted for $\varphi(y)$ as follows:

$$
\begin{array}{lll}
V(y=0, t)=e^{i \omega t} \varphi(0)=\bar{U}_{0} e^{i \omega t} & \rightarrow & \varphi(0)=\bar{U}_{0} \\
V(y \rightarrow \infty, t)=e^{i \omega t} \varphi(y \rightarrow \infty)=0 & \rightarrow & \varphi(y \rightarrow \infty)=0
\end{array}
$$

Here, to solve the N-S, equation (5) is substituted into equation (1) as follows:

$$
i \omega e^{i \omega t} \varphi(y)=v e^{i \omega t} \frac{d^{2} \varphi(y)}{d y^{2}} \quad \rightarrow \quad \frac{d^{2} \varphi(y)}{d y^{2}}-\frac{i \omega \varphi(y)}{v}=0
$$

The ordinary differential equation (8) has solution with two unknown coefficients as follows: 


$$
\varphi(y)=A e^{\sqrt{\frac{i \omega}{v}} y}+B e^{-\sqrt{\frac{i \omega}{v}} y}
$$

In equation (9), the imaginary number $i$ can be written as:

$$
i=e^{i \frac{\pi}{2}} \quad \rightarrow \quad \sqrt{i}=e^{i \frac{\pi}{4}}=\cos \left(\frac{\pi}{4}\right)+i \sin \left(\frac{\pi}{4}\right)=\frac{1}{\sqrt{2}}+\frac{i}{\sqrt{2}}
$$

By combining equations (9) and (10), the solution can be rewritten as follows:

$$
\varphi(y)=A e^{\sqrt{\frac{\omega}{v}} y\left(\frac{1}{\sqrt{2}}+\frac{i}{\sqrt{2}}\right)}+B e^{-\sqrt{\frac{\omega}{v}} y\left(\frac{1}{\sqrt{2}}+\frac{i}{\sqrt{2}}\right)}
$$

where the coefficients A and B are calculated based on boundary conditions in equations (6-7) as:

$$
\begin{array}{lll}
\varphi(y \rightarrow \infty)=0 & \rightarrow & A=0 \\
\varphi(0)=\bar{U}_{0} & \rightarrow & B=\bar{U}_{0}
\end{array}
$$

By substituting values of $A$ and $B$ into equation (11), the function of $\varphi(y)$ is calculated as follows:

$$
\varphi(y)=\bar{U}_{0} e^{-\sqrt{\frac{\omega}{2 \nu}} y(1+i)}
$$

Therefore, the velocity distribution in liquid environment is written as:

$$
V(y, t)=\bar{U}_{0} e^{i \omega t} e^{-\sqrt{\frac{\omega}{2 v}} y(1+i)}=U_{0} e^{-\sqrt{\frac{\omega}{2 v}} y(1+i)}
$$

where $V_{0}$ is the harmonic velocity at the solid/liquid interface. Equation (15) represents a critically damped shear wave radiated into liquid by the oscillating QCM. The decay length is the length in which the generated wave of QCM is transferred within the liquid and is defined through the following equation: 


$$
\delta=\sqrt{\frac{2 v}{\omega}}=\sqrt{\frac{2 \mu}{\rho \omega}}
$$

where $\mu$ and $\rho$ are the viscosity and density of liquid, respectively. Therefore, the solution for velocity distribution in the liquid is written as:

$$
V(y, t)=U_{0} e^{-\frac{y(1+i)}{\delta}}
$$

Based on the velocity distribution in equation (17), the shear stress imposed by the surface on the liquid at the interface is calculated as:

$$
\left.\tau_{y x}\right|_{y=0}=-\left.\mu \frac{d V}{d y}\right|_{y=0}=-\mu U_{0} \frac{-(1+i)}{\delta}\left[e^{\frac{-y(1+i)}{\delta}}\right]_{y=0}=\mu U_{0} \frac{(1+i)}{\delta}
$$

Based on the shear stress, the surface mechanical impedance due to liquid is calculated as ${ }^{1}$ :

$$
Z_{s}=\left.\frac{\tau_{y x}}{U_{0}}\right|_{y=0}=\mu \frac{(1+i)}{\delta}=\mu \frac{(1+i)}{\sqrt{\frac{2 \mu}{\rho \omega}}}=\sqrt{\frac{\rho \mu \omega}{2}}(1+\mathrm{i})
$$

The motional inductance due to liquid $\left(L_{2}\right)$ is calculated as follows ${ }^{1}$ :

$$
L_{2}=\frac{\pi}{4 K^{2} \omega_{s}^{2} C_{0}} \frac{\operatorname{Im}\left(Z_{s}\right)}{Z_{q}}
$$

where $\mathrm{K}$ is the quartz electromechanical coupling coefficient and $C_{0}$ is the static capacitance. The quartz shear-wave characteristic impedance $Z_{q}$ is calculated as follows ${ }^{1}$ :

$$
Z_{q}=\sqrt{\rho_{q} \mu_{q}}
$$

By combining equations (19-21), $L_{2}$ is calculated as:

*Corresponding Author: ho.sun@northeastern.edu 


$$
L_{2}=\frac{\pi}{4 K^{2} \omega_{s}^{2} C_{0}} \frac{1}{\sqrt{\rho_{q} \mu_{q}}} \sqrt{\frac{\rho \mu \omega_{s}}{2}}
$$

The inductance $L_{1}$ due to QCM is calculated as ${ }^{1}$ :

$$
L_{1}=\frac{\pi^{2}}{8 K^{2} \omega_{s}^{2} C_{0}}
$$

Based on parameters $L_{1}$ and $L_{2}$, the frequency shifts of QCM loading with liquid layer with nonslip condition at the interface, is calculated as follows:

$$
\Delta f=-\frac{L_{2}}{2 L_{1}} f_{s}=-f_{s}^{\frac{3}{2}}\left(\frac{\rho \mu}{\pi \rho_{q} \mu_{q}}\right)^{\frac{1}{2}}
$$

Equation (24) is in agreement with the prediction of Kanazawa and Gordon theory ${ }^{2}$.

\section{2) Analytical Study of Frequency Response of QCM with Hydrophobic Smooth Surface}

In this part, an analytical equation for the response of a QCM in liquid in the presence of slip is determined. First, the relationship between the velocities of the solid and the liquid at the interface and the slip length is extracted. Then, the correlation between the response of the QCM and slip length is derived. Figure $2 \mathrm{~S}$ displays the acoustic shear-wave simulation system with the slip length. In this figure, $U_{0}$ and $V_{0}$ are the velocities of the solid and liquid at the interface, respectively.

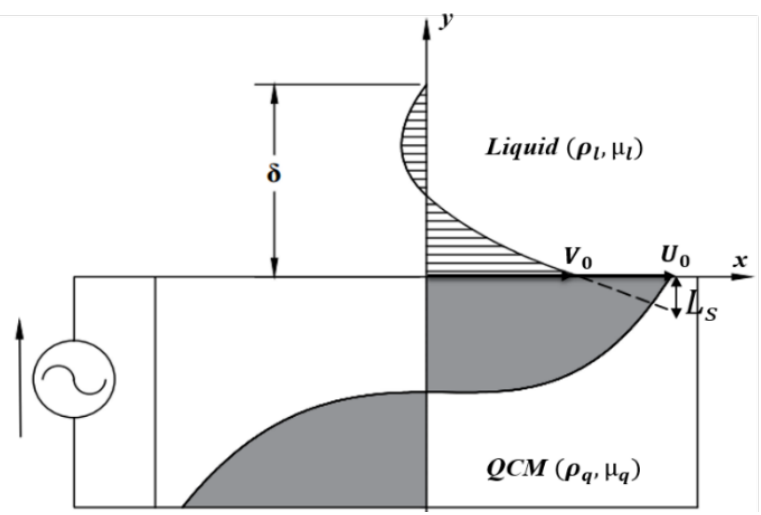

Figure 2S. Illustration of the acoustic shear-wave simulation system with slip length $\mathrm{L}_{s}$. 
Based on the slip velocity $\left(V_{0}-U_{0}\right)$, the stress applied from the solid surface onto the liquid is calculated as follows ${ }^{3}$ :

$$
F_{f}=-\lambda\left(V_{0}-U_{0}\right)
$$

where $\lambda$ is the friction coefficient $(\mathrm{Pa} \cdot \mathrm{s} / \mathrm{m})$. The slip length $\left(L_{s}\right)$ shown in Figure $2 \mathrm{~S}$ is calculated based on the Navier boundary condition ${ }^{4}$ :

$$
L_{s}=\frac{V_{0}-U_{0}}{\left.\frac{d V}{d y}\right|_{y=0}}=\frac{\mu}{\lambda}
$$

The friction force per unit area from solid on liquid is assumed to be equal to the shear stress applying from liquid on solid surface at the interface. Therefore, we will have:

$$
F_{f}=\left.\tau_{y x}\right|_{y=0} \quad \rightarrow \quad-\lambda\left(V_{0}-U_{0}\right)=-\left.\mu \frac{d V}{d y}\right|_{y=0}=\mu V_{0} \frac{(1+i)}{\delta}
$$

By simplifying equation (27), the slip velocity can be written as:

$$
U_{0}-V_{0}=\mu V_{0} \frac{(1+i)}{\delta \lambda}=V_{0} \frac{L_{s}(1+i)}{\delta}
$$

The normalized slip length of $b$ is defined as:

$$
b=\frac{L_{s}}{\delta}
$$

Combining equations (28) and (29), leads to the following equation:

$$
U_{0}-V_{0}=V_{0} b(1+i) \quad \rightarrow \quad V_{0}=\frac{U_{0}}{1+b(1+i)}
$$


From the above equation, if the slip length $L_{s}$ is much smaller than the decay length $(\delta), U_{0}=V_{0}$ which corresponds to the non-slip condition. By combining equations (18) and (30), the shear stress in presence of slip length at the interface is calculated as:

$$
\left.\tau_{y x}\right|_{y=0}=-\left.\mu \frac{d V}{d y}\right|_{y=0}=\mu \frac{U_{0}(1+i)}{\delta(1+b+i b)}=\mu \frac{U_{0}(1+i)}{\delta(1+b+i b)} \frac{(1+b-i b)}{(1+b-i b)}=\mu \frac{U_{0}(1+2 b+i)}{\delta\left(1+2 b+2 b^{2}\right)}
$$

Similar to equation (19), the surface mechanical impedance due to liquid is calculated as follows:

$$
Z_{s}=\left.\frac{\tau_{y x}}{U_{0}}\right|_{y=0}=\mu \frac{(1+2 b+i)}{\delta\left(1+2 b+2 b^{2}\right)}
$$

By substituting equation (32) into equation (20), the motional inductance due to liquid $L_{2}$ is calculated as follows:

$$
L_{2}=\frac{\pi}{4 K^{2} \omega_{s}^{2} C_{0}} \frac{\operatorname{Im}\left(Z_{s}\right)}{Z_{q}}=\frac{\pi}{4 K^{2} \omega_{s}^{2} C_{0}} \frac{1}{\sqrt{\rho_{q} \mu_{q}}} \frac{\mu}{\sqrt{\frac{2 \mu}{\rho \omega}}\left(1+2 b+2 b^{2}\right)}
$$

By combining equations (23) and (33), the frequency shifts of the QCM loading with liquid layer in the presence of slip condition at the interface, is calculated as follows ${ }^{3}$ :

$$
\Delta f=-\frac{L_{2}}{2 L_{1}} f_{s}=-f_{s}^{\frac{3}{2}}\left(\frac{\rho \mu}{\pi \rho_{q} \mu_{q}}\right)^{\frac{1}{2}} \frac{1}{1+2 b+2 b^{2}}
$$

If the value of non-dimensional slip length $b$ is zero, equation (34) will be corresponding to the Kanazawa and Gordon theory in equation (24). The equation (34) is employed to obtain the slip length based on the experimental measurement of frequency shift of the QCM in liquid.

\section{3) Analytical Drag Reduction Measurement using QCM}

In this part, the drag reduction on QCM in presence of slip boundary condition is determined. To do this, the shear stresses at solid/liquid interface for non-slip and slip conditions, which were described earlier in equations (18) and (31), are employed. Based on these equations, Drag Reduction (DR) is calculated as follows:

*Corresponding Author: ho.sun@northeastern.edu 


$$
\begin{aligned}
& D R=\frac{\left(\left.\tau_{y x, \text { slip }}\right|_{y=0} \cdot A\right)-\left(\left.\tau_{y x, \text { no slip }}\right|_{y=0} \cdot A\right)}{\left.\tau_{y x, \text { no slip }}\right|_{y=0} \cdot A}=\frac{\left.\tau_{y x, \text { slip }}\right|_{y=0}}{\left.\tau_{y x, \text { no slip }}\right|_{y=0}}-1 \\
& D R=\frac{\mu \frac{U_{0}(1+2 b+i)}{\delta\left(1+2 b+2 b^{2}\right)}}{\mu U_{0} \frac{(1+i)}{\delta}}-1=\frac{(1+2 b+i)}{\left(1+2 b+2 b^{2}\right)(1+i)}-1=\frac{1+b-i b}{1+2 b+2 b^{2}}-1 \\
& D R=\frac{-b(1+2 b+i)}{1+2 b+2 b^{2}}
\end{aligned}
$$

where $b$ is the normalized slip length defined in equation (29). Since we assumed that the velocity profile is one-dimensional in the $x$-direction, the DR is determined using the real part of equation (37) as follows:

$$
D R=\frac{-b(1+2 b)}{1+2 b+2 b^{2}}
$$

Equation (38) describes that DR is only function of non-dimensional slip length $b$.

\section{4) Epoxy-Based Superhydrophobic Coating on QCM}

The details of the fabrication procedure are described as follows as shown in Figure 3S:

\section{Step 1) Coating Solution Preparation}

The part A and part B of epoxy (Epo-tek 301, Epoxy Technology) with a ratio of 4:1 was mixed in a container and the mixture stirred for 2 minutes by a gentle mechanical stirring. 1.5 gr fumed silica nanoparticles (Cab-o-sil TS-720, Cabot Corp.) and 28.5 gr fluorinated alkyl silane isopropanol solution (Dynasylan ${ }^{\circledR} \mathrm{F}-8263$, Evonik industries) were then mixed in a vial (Vial 1) and stirred on a magnetic stirrer plate for 20 minutes. 6 gr epoxy, 24 gram acetone (ACS reagent, $\geq 99.5 \%$, Sigma Aldrich), $0.12 \mathrm{~g}$ (3-glycidoxypropyl) triethoxysilane (Gelest Inc.) and $0.012 \mathrm{~g} \mathrm{~N}$, N'-bis [3-(trimethoxysilyl) propyl] ethylenediamine (Gelest Inc.) were mixed in another vial (Vial 2) and stirred for 20 minutes. At last, the solution in the Vial 1 was poured into Vial 2 while stirring for another 1 hour.

\section{Step 2) Spray Coating}

A high volume low pressure (HVLP) spray gun (DeVilbiss 802342 Starting Line HVLP Gravity Spray Gun) was used to spray-coat the solution on the QCM substrates. The spray pressure was set to $2 \times 10^{5} \mathrm{~Pa}$ and the spray gun was kept $20 \mathrm{~cm}$ away from the substrate at all times and moving at a speed of $7 \mathrm{~cm} / \mathrm{s}$ horizontally. The spray was repeated on each QCM substrate for 3 times.

\footnotetext{
*Corresponding Author: ho.sun@northeastern.edu
} 


\section{Step 3) Curing}

After the spray coating was completed, the samples were cured in a $110^{\circ} \mathrm{C}$ oven for two hours. Finally, the samples were removed from the oven and cooled in air.
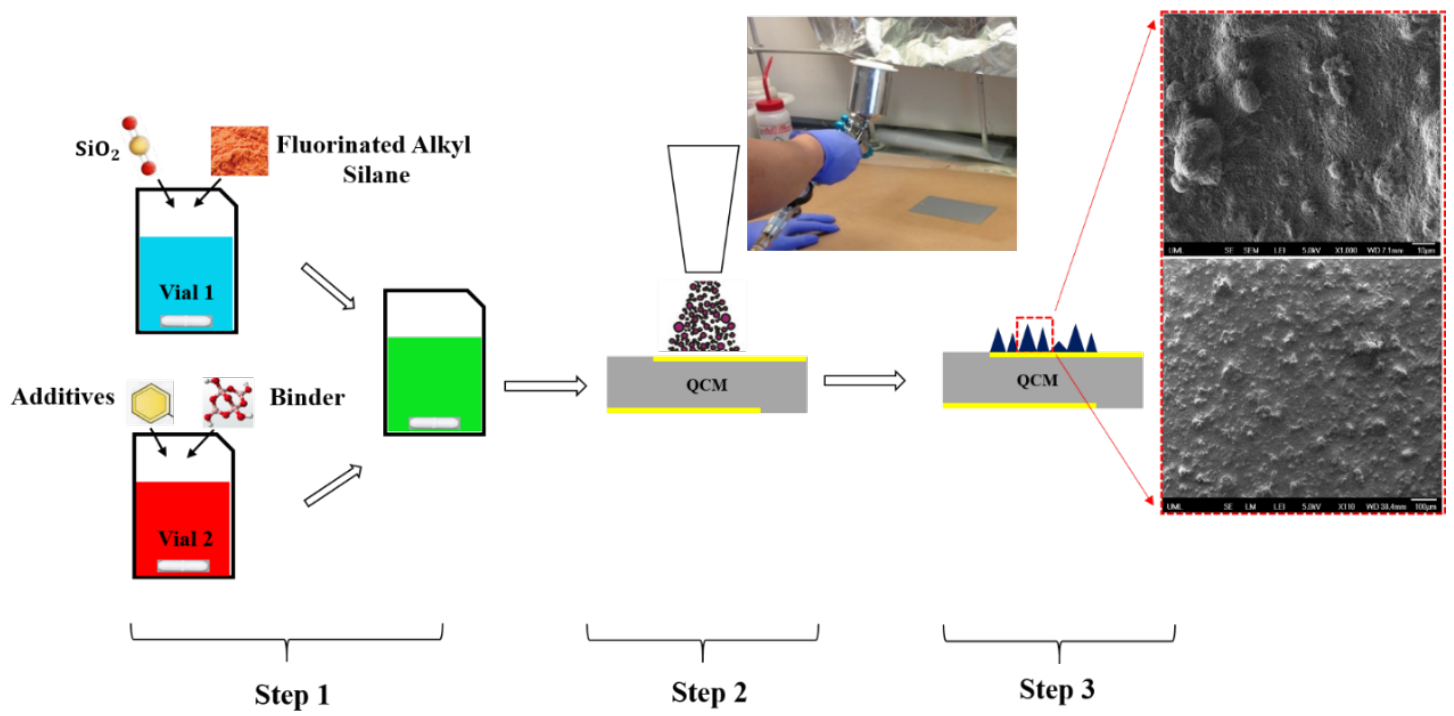

Figure 3S. Fabrication procedure of random superhydrophobic coating on QCM substrate (Insets: SEM images of superhydrophobic coating on a QCM substrate).

\section{5) PMMA Micropillar-Based Superhydrophobic Coating on QCM}

The details of the fabrication procedure are illustrated in Figure $4 \mathrm{~S}$, including a five-step procedure as follows:

\section{Step 1) SU-8 Mold Preparation}

SU-8 is a common negative photoresist in Microelectromechanical systems (MEMS) fabrication. The "negative" means that the parts exposed to UV light become hard whereas the unexposed area can be washed away during the development procedure [191]. Here, an SU-8 mold was fabricated utilizing a UV photolithography technique (Mask Aligner, Suss MicroTec MA6).

\section{Step 2) PDMS Mold Preparation}

Polydimethylsiloxane (PDMS) is a common and inexpensive elastomer that is utilized in various types of microfluidic devices ${ }^{5}$. Here, a PDMS stamp was fabricated using traditional soft lithography which matched the geometry of the SU-8 mold.

\section{Step 3) Spin Coating of PMMA Solution}

PMMA and 2-ethoxyethyl acetate solvent were mixed (20\% PMMA by weight) and heated to $50{ }^{\circ} \mathrm{C}$ with overnight stirring. The resulting PMMA solution was dispensed onto the PDMS stamps with microholes and then spin-coated at $5000 \mathrm{rpm}$ for 60 seconds to enable 
the PMMA solution to fill into the microholes. Then, the PDMS stamp with PMMA solution was heated on a hot plate at $100{ }^{\circ} \mathrm{C}$ for 5 minutes to evaporate the solvent.

\section{Step 4) Fabrication of Micropillars using T-NIL}

The PDMS mold with the PMMA was inverted and pressed onto the QCM under high temperature using T-NIL (NX-2600, Nanonex Corp., NJ). Later, the PDMS stamp was released by peeling from the QCM and only the PMMA micropillar was left on the QCM substrate.

\section{Step 5) Superhydrophobic Coating Treatment using CVD}

PMMA as a hydrophobic material has $-\mathrm{C}-\mathrm{H}$ bonds on its surface. After the fabrication of micropillar structures on the QCM, the entire surface including tips and side walls of the micropillars were treated with trichloro $(1 \mathrm{H}, 1 \mathrm{H}, 2 \mathrm{H}, 2 \mathrm{H}$-Perfluorooctyl) silane (PFOTS) in a vacuum desiccator by CVD technique at room temperature for 24 hours. The deposited layer contains $-\mathrm{C}-\mathrm{F}$ bonds which give the surface superhydrophobic property ${ }^{6}$.

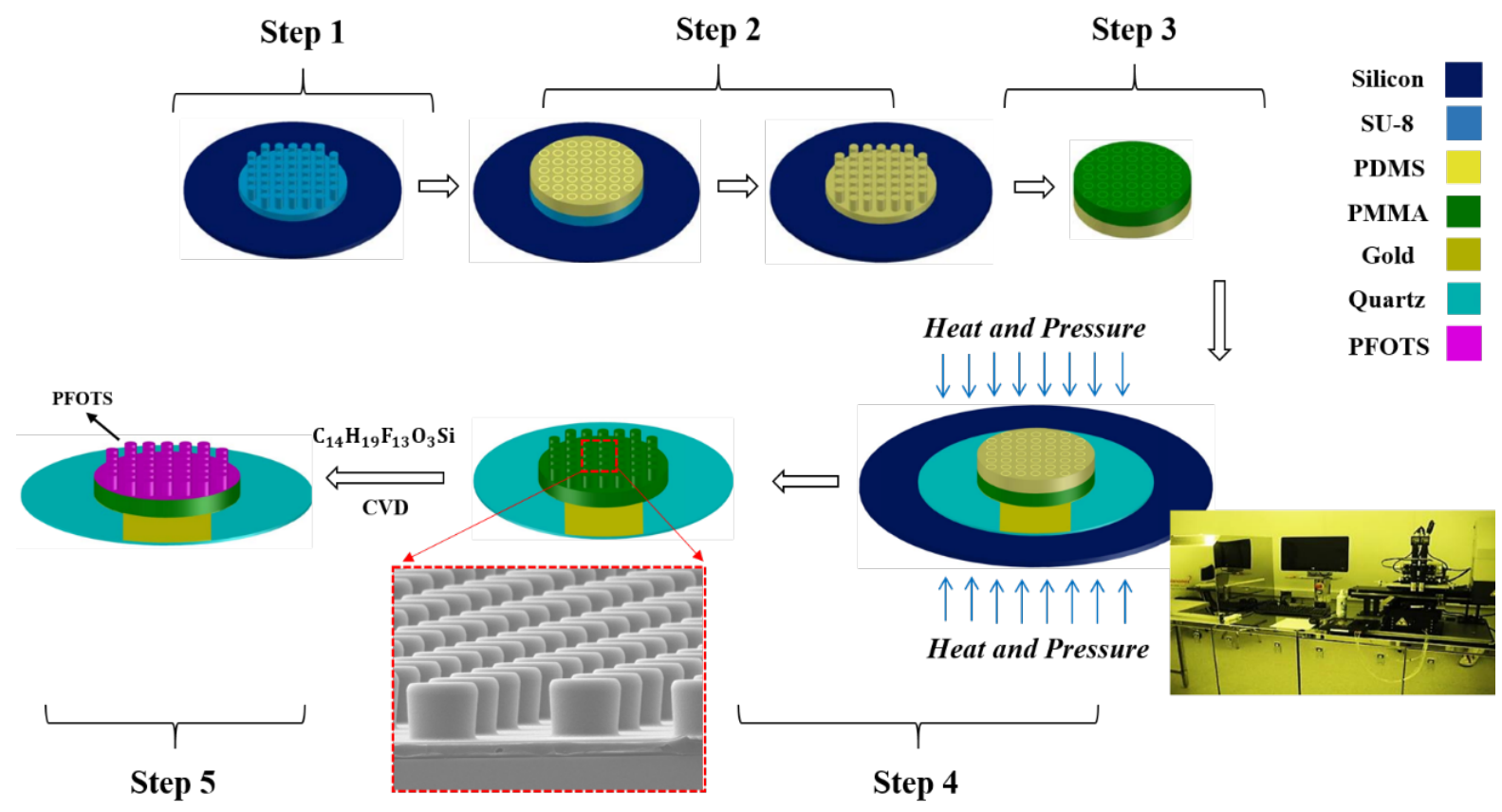

Figure 4S. Fabrication procedure of micropillar structures with superhydrophobic state on the bare QCM using T-NIL and CVD techniques.

\section{6) Analytical Model for Drag Reduction Measurement using Rheometer}

In the ARES-G2 rheometer, the top plate is stationary, and the bottom plate is rotating with a constant velocity $\left(\mathrm{U}_{\text {plate }}\right.$ ) as shown in Figure $5 \mathrm{~S}(\mathrm{a})$. The velocity profile between two parallel plates in the rheometer for non-slip and slip conditions are shown in Figure $5 S(b-c)$. 


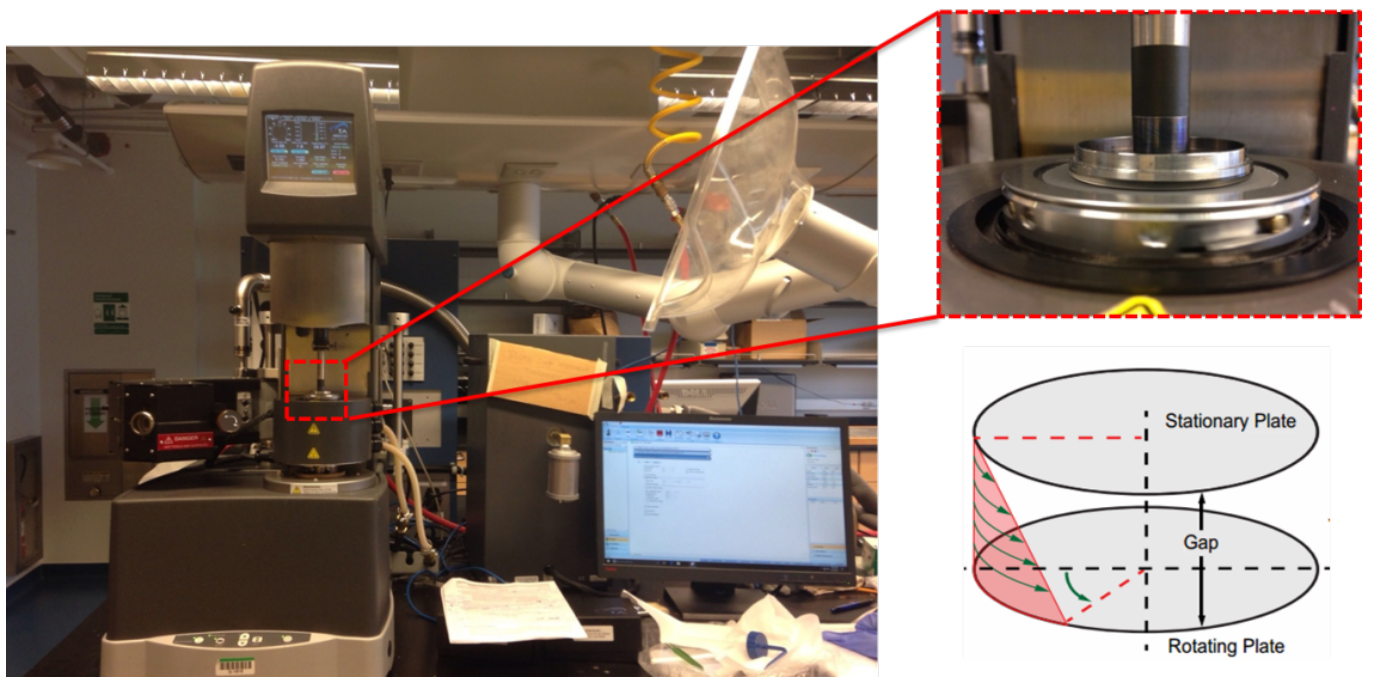

(a)

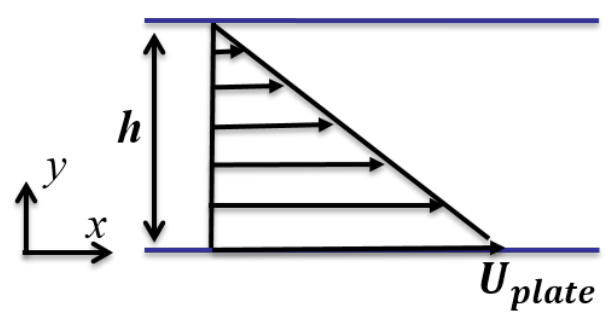

Nonslip Condition

(b)

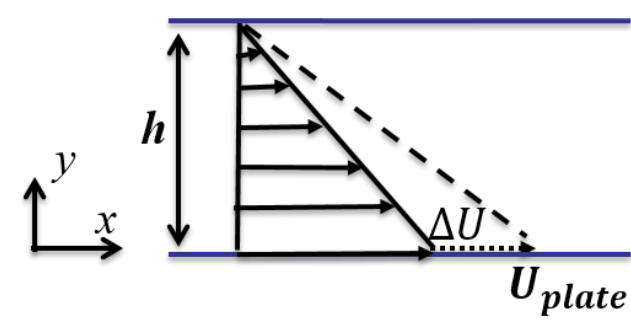

Slip Condition

(c)

Figure 5S. (a) ARES G-2 rheometer and velocity profiles between two parallel plates for (b) non-slip and (c) slip conditions.

The velocity profile for non-slip condition is linear which is calculated as follows:

$$
u_{1}(y)=U_{\text {plate }}-\frac{U_{\text {plate }}}{h} y
$$

where $\mathrm{h}$ is the gap size between two parallel plates. For the bottom surface with superhydrophobic coating, there is a slip velocity of $\Delta U$ as shown in Figure $5 S(c)$. Therefore, the velocity profile is calculated as follows:

$$
u_{2}(y)=U_{\text {plate }}-\Delta U-\frac{U_{\text {plate }}-\Delta U}{h} y
$$


If the slip velocity is $\Delta U=0$, the velocity profile in equation (40) will be the same as for the nonslip condition in equation (39). The drag reduction for such a surface with slip velocity in comparison to non-slip condition is calculated as follows:

$$
\text { Drag Reduction }=\frac{F_{\text {Slip }, y=0}-F_{\text {noslip }, y=0}}{F_{\text {noslip }, y=0}}=\frac{F_{\text {Slip }, y=0}}{F_{\text {noslip }, y=0}}-1=\frac{\left(\tau_{\text {Slip }, y=0}\right)(A)}{\left(\tau_{\text {noslip }, y=0}\right)(A)}-1
$$

Based on the velocity profile in equations (39) and (40), the shear stresses at solid/liquid interface for non-slip and slip conditions are calculated as follows:

$$
\begin{aligned}
& \tau_{\text {noslip } y=0}=\left.\mu \frac{d u_{1}}{d y}\right|_{y=0}=-\mu \frac{U_{\text {plate }}}{h} \\
& \tau_{\text {Slip }, y=0}=\left.\mu \frac{d u_{2}}{d y}\right|_{y=0}=-\mu \frac{U_{\text {plate }}-\Delta U}{h}
\end{aligned}
$$

where $\mu$ is the viscosity of liquid. Based on the shear stresses, the drag reduction is calculated using the slip velocity as follows:

$$
\text { Drag Reduction }=\frac{-\mu \frac{U_{\text {plate }}-\Delta U}{h}}{-\mu \frac{U_{\text {plate }}}{h}}-1=-\frac{\Delta U}{U_{\text {plate }}}
$$

In order to measure the slip velocity experimentally, torque measurements via rheometer are used. The torque values for non-slip conditions are calculated as follows:

$$
T_{\text {no slip }}=\int_{0}^{R} r\left(\tau_{\text {no slip }}\right)(2 \pi r) d r=\int_{0}^{R} 2 \pi r^{2} \tau_{\text {no slip }} d r=\int_{0}^{R} 2 \pi r^{2} \mu \frac{r \omega}{h} d r=\frac{\pi \mu \omega R^{4}}{2 h}
$$

where $R$ is the radius of the plate and $\omega$ is the angular velocity. For the bottom plate with superhydrophobic coating, the torque is written as follows:

$$
T_{\text {Slip }}=\int_{0}^{R} r\left(\tau_{\text {Slip }}\right)(2 \pi r) d r=\int_{0}^{R} 2 \pi r^{2} \tau_{\text {Slip }} d r=\int_{0}^{R} 2 \pi r^{2} \mu \frac{r \omega-\Delta U}{h} d r
$$




$$
T_{\text {Slip }}=\frac{2 \pi \mu \omega}{h} \int_{0}^{R} r^{3} d r-\frac{2 \pi \mu}{h} \int_{0}^{R} r^{2} \Delta U d r=\frac{\pi \mu \omega R^{4}}{2 h}-\frac{2 \pi \mu}{h} \int_{0}^{R} r^{2} \Delta U d r
$$

where the second term appears due to the slip velocity. In order to get rid of the integral in the second component, it is necessary to find a correlation for slip velocity. Since the slip velocity has relationships with distance $(r)$ and angular velocity $(\omega)$, it can be expressed as:

$$
\Delta U \propto r \text { and } \omega \quad \rightarrow \quad \Delta U=K r \omega
$$

where $\mathrm{K}$ is a non-dimensional constant. By combing equations (46) and (47), the torque for slip condition can be written as follows:

$$
T_{\text {Slip }}=\frac{\pi \mu \omega R^{4}}{2 h}-\frac{\pi \mu K \omega R^{4}}{2 h}=T_{\text {noslip }}-\frac{\pi \mu K \omega R^{4}}{2 h}
$$

Comparing equations (48) and (45), the ratio of torque for slip condition to that of non-slip condition can be written as:

$$
\frac{T_{\text {Slip }}}{T_{\text {no slip }}}=1-K
$$

Combining equations (44) and (49), the drag reduction is calculated based on values of torques as:

$$
\text { Drag Reduction }=-\frac{\Delta U}{U_{\text {plate }}}=-\frac{\Delta U}{r \omega}=-\frac{K r \omega}{r \omega}=-K=\frac{T_{\text {Slip }}-T_{\text {noslip }}}{T_{\text {noslip }}}
$$

\section{7) Experimental Setup for QCM Measurement}

The $10 \mathrm{MHz}$ AT-cut QCMs were purchased from FORTIMING Corporation (Marlboro, MA, USA) and have a blank diameter of $13.7 \mathrm{~mm}$, electrode diameter of $5.11 \mathrm{~mm}$ and quartz thickness of $0.17 \mathrm{~mm}$. The electrode was polished and has a $100 \AA$ thickness of gold layer on a titanium adhesive layer. To measure the QCM frequency shift, an experimental setup consisting of a network analyzer (HP8753C and 85046-A S-Parameter test set), a data acquisition (DAQ) system, a built-in LabVIEW program (National Instruments) and the QCM device was built. The network analyzer actuates the QCM device, while recording the reflected signal created by the QCM 
device. Finally, the resonant frequency was directly measured with the LabVIEW program. Figure 6s displays the experimental setup used for QCM measurement.

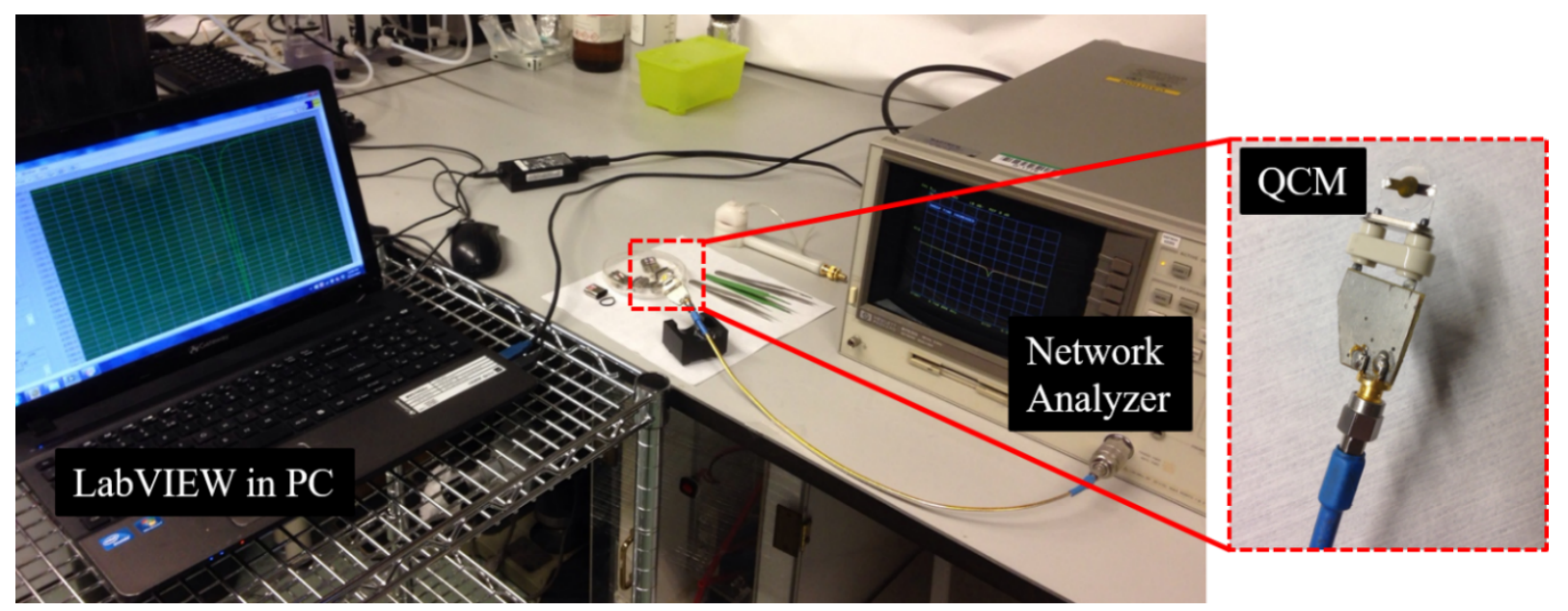

Figure 6s. Experimental setup for QCM measurement system.

\section{8) Density and Viscosity of Aqueous Sugar/Water Solutions}

Table 1S lists information for density and viscosity of aqueous sugar/water solutions for different concentrations.

Table 1S. Density and viscosity of sugar/water solutions for different concentrations.

\begin{tabular}{|c|c|c|}
\hline Concentration (\%) & Density $\left(\mathbf{k g} / \mathbf{m}^{\mathbf{3}}\right)$ & Viscosity (mPa.s) \\
\hline 0 & 1000 & 1.50 \\
10 & 1038 & 1.59 \\
20 & 1081 & 1.74 \\
30 & 1127 & 3.0 \\
40 & 1177 & 5.31 \\
50 & 1230 & 15.0 \\
60 & 1300 & 45.0 \\
\hline
\end{tabular}

\section{References}

1. Ballantine, D. S.; White, R. M.; Ricco, A. J.; Zellers, E. T.; Frye, G. C.; Wohltjen, H., Acoustic Wave Sensors: Theory, Design, \& Physico-Chemical Applications. 1 ed.; Academic Press: 1997, Chapter 3, 36-149. 2. Kanazawa, K. K.; Gordon, J. G., Frequency of a quartz microbalance in contact with liquid. Analytical Chemistry 1985, 57 (8), 1770-1771.

3. Huang, K.; Szlufarska, I., Friction and Slip at the Solid/Liquid Interface in Vibrational Systems. Langmuir 2012, 28 (50), 17302-17312.

4. Navier, C. L. M. H., Mémoire sur les lois du mouvement des fluides: lu à l'Académie Royale des Sciences ; le 18 mars 1822. 1822, 389-416. 
5. Oh, S. R., Thick single-layer positive photoresist mold and poly(dimethylsiloxane) (PDMS) dry etching for the fabrication of a glass-PDMS-glass microfluidic device. Journal of Micromechanics and Microengineering 2008, 18 (11), 115025, 1-10.

6. Wang, P.; Su, J.; Shen, M.; Ruths, M.; Sun, H., Detection of Liquid Penetration of a Micropillar Surface Using the Quartz Crystal Microbalance. Langmuir 2017, 33 (2), 638-644.

*Corresponding Author: ho.sun@northeastern.edu 